\title{
Comparisons of short-term outcomes between robot-assisted and thoraco-laparoscopic esophagectomy with extended two-field lymph node dissection for resectable thoracic esophageal squamous cell carcinoma
}

\author{
Junying Chen ${ }^{1,2 \#}$, Qianwen Liu ${ }^{1,2 \#}$, Xu Zhang ${ }^{1,2}$, Hong Yang ${ }^{1,2}, Z_{\text {Zihui Tan }}^{1,2}$, Yaobin Lin ${ }^{1,2}$, Jianhua Fu ${ }^{1,2}$ \\ ${ }^{1}$ Sun Yat-sen University Cancer Center; State Key Laboratory of Oncology in South China; Collaborative Innovation Center for Cancer Medicine, \\ Guangzhou 510060, China; ${ }^{2}$ Guangdong Esophageal Cancer Institute, Guangzhou 510060, China \\ Contributions: (I) Conception and design: J Chen, Q Liu, J Fu; (II) Administrative support: X Zhang, H Yang; (III) Provision of study materials or \\ patients: X Zhang, H Yang; (IV) Collection and assembly of data: J Chen, Z Tan, Y Lin; (V) Data analysis and interpretation: J Chen, Q Liu; (VI) \\ Manuscript writing: All authors; (VII) Final approval of manuscript: All authors. \\ \#These authors contributed equally to this work. \\ Correspondence to: Jianhua Fu. Department of Thoracic Surgery, Sun Yat-sen University Cancer Center, State Key Laboratory of Oncology in South \\ China, Guangdong Esophageal Cancer Institute, Collaborative Innovation Center for Cancer Medicine, Guangzhou 510060, China. \\ Email: fujianhua2014@yahoo.com.
}

Background: Video-assisted thoracoscopic surgery has been identified as priori choice compared with open approaches in esophageal cancer surgery. With the developments in the Da Vinci robotic system, the robot-assisted minimally invasive esophagectomy (RAMIE) has been increasingly popular. However, whether RAMIE could be a better choice over thoraco-laparoscopic minimally invasive esophagectomy (TLMIE) is unclear.

Methods: The clinicopathological characteristics of patients who received RAMIE or TLMIE with modern two-field lymph node dissection in Sun Yat-sen University Cancer Center between Jan 2016 to Jan 2018 were retrospectively retrieved. The 1:1 propensity score match analysis was performed to compare the shortterm effectiveness and safety between the two groups.

Results: Two hundred and fifteen esophageal squamous cell carcinoma (ESCC) patients received RAMIE (101 patients) or TLMIE (114 patients) were included in the analysis. After a 1:1 propensity score matching, 108 patients (54 pairs) who received RAMIE or TLMIE displayed no significant variance in baseline clinicopathological characteristics. No significant difference in operative time, intraoperative blood loss, number of resected lymph nodes, and R0 resection rates were observed between the matched groups. However, the recurrent laryngeal nerve protection was better in RAMIE group $(\mathrm{P}=0.021)$. Nevertheless, both the incidences of common postoperative complications and length of ICU (hospital) stay were similar in two groups. The average total $(\mathrm{P}=0.009)$ and daily $(\mathrm{P}=0.028)$ expenses of RAMIE were higher.

Conclusions: In general, RAMIE could benefit patients by providing better recurrent laryngeal nerve protection. In order to promote the applications of RAMIE, more efforts should be made to reduce the costs by the social and medical insurance agencies.

Keywords: Esophageal cancer; thoracoscopy; laparoscopy; robot-assisted esophagectomy

Submitted Mar 16, 2019. Accepted for publication Aug 27, 2019.

doi: $10.21037 /$ jtd.2019.09.05

View this article at: http://dx.doi.org/10.21037/jtd.2019.09.05 


\section{Introduction}

Esophagectomy with extended lymphadenectomy remains a mainstay of curative treatment for patients with localized esophageal squamous cell carcinoma (ESCC). The traditional open transthoracic procedure is the standard surgery for esophageal cancer (1-4). However, the videoassisted thoracoscopic surgery, with less surgical trauma, blood loss, postoperative complications, and shorter hospital stay, has been gradually recognized as a more feasible surgical procedure for esophageal cancer (3,5-7). Also, the long-term oncologic outcomes of thoraco-laparoscopic minimally invasive esophagectomy (TLMIE) were found to be equivalent to standard open surgery $(4,8,9)$. However, the TLMIE is limited by a two-dimensional view and the reduced freedom of movements, which may disable delicate dissections of the esophagus and lymph nodes, especially in salvage surgery after definitive chemoradiotherapy or surgery following neo-adjuvant therapies.

With the recent developments in surgical instruments, robot-assisted minimally invasive esophagectomy (RAMIE) performing with the Da Vinci Si robotic system has an increasing usage throughout the world (10-15). Comparing with TLMIE, RAMIE offers advantages including tremor filtration, three-dimensional visualization, ten-timesenlarged image and seven-degree freedom of its dexterity endowrists, all of which provide a stable and comfortable environment for the surgeon to operate. van Hillegersberg et al. reported a $90 \% \mathrm{R} 0$ resection rate in ten downstaged cT4b ESCC patients with RAMIE, due to the enlarged 3D image allowing for very precise dissection of the irradiated tumor tissue from the trachea, bronchi, and aorta (16).

Nonetheless, whether RAMIE has comparable impacts with TLMIE on the short-term surgical outcomes of ESCC is unclear. Therefore, we performed a propensity score matched (PSM) study to objectively compare the surgical results between TLMIE and RAMIE.

\section{Methods}

\section{Patients}

This study was approved by the institutional review board of Sun Yat-sen University Cancer Center, and the patients' consent was waived. From January 2016 to Jan 2018, a total of 215 patients received minimal invasive esophagectomy and lymphadenectomy by the same surgical team in the thoracic surgery department of Sun Yat-sen University Cancer Center, Guangzhou, China. The inclusion criteria are: (I) patients had primary and histologically identified squamous cell carcinoma; (II) patients received RAMIE or TLMIE with gastric pull-up and cervical anastomosis; (III) patients received extend two-field lymphadenectomies.

The exclusion criteria are: (I) patient had a history of other malignant diseases; (II) patient records lacked sufficient general, pathological, and neo-adjuvant therapeutic information for analysis, i.e., age, gender, tumor locations, pathological stages, regimens of neo-adjuvant treatments.

\section{Preoperative workup}

Preoperative tumor stage evaluations included history taking, physical examination, chest and upper abdominal contrast computed tomography (CT) and ultrasonography or CT of the neck, endoscopic ultrasonography of upper gastrointestinal tract and pathological biopsy. Endobronchial ultrasonography was routinely taken in patients with middle thoracic esophageal lesions.

\section{Surgical approaches}

In RAMIE, three-arm technique is used in both thoracic and abdominal procedures of McKeown esophagectomy. The detail techniques were described elsewhere (17). The enteral tube is placed through a percutaneous jejunostomy. We did the esophagogastric stapled anastomosis in the neck. A drain is placed $3 \mathrm{~cm}$ below the neck wound with inner tip beside the anastomosis, and all incisions are closed.

In TLMIE, the McKeown procedures are applied, as well. The patient position and procedure steps inside the thoracic, abdominal cavity and the neck anastomosis are same in RAMIE and TLMIE.

\section{Postoperative management}

Patients are extubated approximately one hour after surgery, before transferred from the resuscitation room to the intensive care unit. On postoperative day one, the nasogastric tubes are routinely removed if the drainage is less than $100 \mathrm{~mL}$ and the color does not indicate hemorrhage. Then, the patients will be transferred to the general surgical ward on postoperative day two, if their vital signs are stable. All patients are fed through the enteral tube during the postoperative day two to seven. On day seven, the $\mathrm{x}$-ray barium radiography is performed routinely to check for anastomosis leakage. Patients will be able to start 
with sips of water without signs of anastomotic dehiscence, and the oral intake is gradually return to normal solid food within 14 days after the operation.

\section{Statistical analysis}

The pathological stages were identified according to the 7th edition of the American Foint Committee on Cancer Staging Manual. The clinicopathological characteristics between the two groups were compared using Student's $t$-test and the Chi-square tests. Statistical analysis performed using SPSS 23.0 (SPSS Corporation, Chicago, IL, USA). Significance was set to $\mathrm{P}<0.05$.

The 1:1 PSM was performed using characteristics including age, gender, BMI, history of smoking or alcohol consumption, hypertension, diabetes, arrhythmia, coronary heart disease, clinical $\mathrm{T}, \mathrm{N}$ stage and neoadjuvant chemoradiotherapy. The regression model used for calculating propensity score was $\kappa-$ Nearest neighbors matching. The standardized difference in means were compared before and after matching to evaluate covariate balance between two groups (18). The PSM was performed by the "psmatch2" command in STATA 12.0 software.

\section{Results}

\section{Patient characteristics}

A total of 215 patients undertook minimal invasive esophagectomy and lymphadenectomy by the same surgical team between January 2016 and January 2018. According to the inclusion and exclusion criteria, 68 patients were excluded because they had adenocarcinoma of esophagogastric junction; four patients were excluded for synchronous multiple primary cancers; and one patient was excluded because of having intrathoracic anastomosis. Therefore, a total of 142 patients (68 and 74 in the RAMIE and TLMIE group, respectively) were enrolled in the study.

In the study cohort, the majority were male $(112 / 142$, $78.9 \%)$ patients. The median age of the cohort was 62 years (range, $43-84$ year). Seventy-four patients $(52.1 \%)$ had the history of smoking ( $\geq 20$ pack-years), and 30 (21.1\%) had alcohol assumption history. Postoperative pathological staging revealed that $31(21.8 \%)$ cases were in stage I, $51(35.9 \%)$ in stage II, $40(28.2 \%)$ in stage III. Fortyfive $(31.7 \%)$ patients in the cohort received neoadjuvant chemoradiotherapy, and the pathological complete response rate was $44.4 \%$ (20/45). The demographic characteristics of the study cohort are displayed in Table 1.

The 1:1 PSM was performed to balance the confounding factors between two groups. For the continuous variables, the standardized difference in means were calculated to evaluate the matching balance. Before matching, the standardized difference in mean age and BMI were -0.008 and 0.003 . Whereas the data were 0 and 0.007 respectively for age and BMI after matching. Additionally, the P values did not reveal statistical difference in both the independent t-test of continuous variables and the Chi-square test of binary variables after matching (Table 1).

\section{Comparisons of short-term outcomes in RAMIE and TLMIE group}

The incidence of surgical complications in the entire cohort included cervical anastomotic leakage (8/142, 5.6\%), recurrent laryngeal nerve injury (31/142, $21.8 \%)$, and chylothorax $(5 / 142,3.5 \%)$. All anastomotic leaks of patients were minor leakages that restricted around the cervical anastomosis, without septicemia. The treatment was a prolongation of enteral feeding (up to 36 days after surgery) until no leakage could be identified in gastroscopy. The chylothorax was also treated conservatively, indicating that the low-volume leakage might be from side branches of the thoracic duct; and the vocal cord paralysis was temporary and was able to recover within six months after surgery.

Non-surgical complications included pneumonia (29/142, 20.4\%), ARDS (5/142, 3.5\%), and cardiac events $(2 / 142,1.4 \%)$. The pneumonia was diagnosed and treated with sensitive antibiotics; ARDS were all cured by extended ventilation in the ICU, and the two patients with cardiac events had temporary supraventricular tachycardia and acute right-sided heart failure, respectively.

The radical resection (R0) was achieved in 141 (99.3\%) patients. The patient who did not receive $\mathrm{R} 0$ resection had ESCC located between 28 to $33 \mathrm{~cm}$ from incisors. The esophagus is cut in the thoracic inlet routinely, which should be qualified to have adequate margin in this case. However, the postoperative pathological examination found squamous carcinoma cells in the upper margin. It is possible that the patient had multiple ESCCs in the esophagus, but the second tumor site was too early in stage to be identified by NBI endoscopy.

The mean number of dissected lymph nodes was 26.2 , ranging from 8 to 68 . No reoperation or postoperative death happened within 30 days after an esophagectomy. 
Table 1 Patient characteristics in RAMIE and TLMIE groups before and after matching

\begin{tabular}{|c|c|c|c|c|c|c|}
\hline Variables & \multicolumn{3}{|c|}{ Unmatched cases } & \multicolumn{3}{|c|}{ Matched cases } \\
\hline Age & $61.3 \pm 8.2$ & $61.9 \pm 8.5$ & 0.935 & $61.8 \pm 8.3$ & $61.8 \pm 9.4$ & 0.991 \\
\hline Gender (M/F) & $59 / 15$ & $53 / 15$ & 0.794 & $43 / 11$ & $41 / 13$ & 0.643 \\
\hline Smoking $(\mathrm{Y} / \mathrm{N})$ & $35 / 39$ & $37 / 37$ & 0.397 & $27 / 27$ & $25 / 29$ & 0.700 \\
\hline BMI & $23.0 \pm 3.1$ & $22.8 \pm 2.8$ & 0.657 & $23.0 \pm 2.7$ & $22.7 \pm 2.9$ & 0.548 \\
\hline Hypertension (\%) & $12(16.2)$ & $9(13.2)$ & 0.695 & 7 (13.0) & $8(14.8)$ & 0.781 \\
\hline Diabetes (\%) & $1(1.4)$ & $2(2.9)$ & 0.595 & $1(1.9)$ & $1(1.9)$ & 1.000 \\
\hline Arrhythmia (\%) & $2(2.7)$ & $1(1.5)$ & 1.000 & $2(3.7)$ & $1(1.9)$ & 1.000 \\
\hline cT stage (T1/2/3/4a) & $23 / 9 / 41 / 1$ & $16 / 8 / 44 / 0$ & 0.570 & $15 / 7 / 31 / 1$ & $14 / 7 / 33 / 0$ & 0.778 \\
\hline cN stage (N0/1/2/3) & $37 / 19 / 16 / 2$ & $36 / 19 / 11 / 2$ & 0.903 & $22 / 14 / 16 / 2$ & $30 / 11 / 11 / 2$ & 0.472 \\
\hline
\end{tabular}

RAIME, robot-assisted minimally invasive esophagectomy; TLMIE, thoraco-laparoscopic minimally invasive esophagectomy; BMI, body mass index; CHD, coronary heart disease; nCRT, neo-adjuvant chemoradiotherapy.

The median length of intensive care unit stay was one day (range, 1-36 days), and median postoperative hospital stay was 13 days (range, 3-72 days).

Before matching, more patients in the RAMIE group received nCRT (Table $1, \mathrm{P}=0.020$ ). The incidences of postoperative complications, such as hoarseness, respiratory complications and anastomosis leakages were higher in TLMIE group, but without any statistical difference; however, the average costs for patients received RAMIE were significantly increased (Table $2, \mathrm{P}=0.002$ ).

After the matching process, 108 patients were included in the analysis. Nevertheless, in the matched comparisons, the incidence of vocal cord paralysis was prominently higher in the TLMIE group ( $\mathrm{P}=0.021)$, suggesting the benefit of recurrent laryngeal nerve protection comes with RAMIE. Both the total expenses and daily expenses of RAMIE was higher than TLMIE, though the difference was less significant after matching (Table 2).

\section{Discussion}

As RAMIE becoming more popular, it is significant to explore the procedure-related safety and applicability issues. In our study, TLMIE and RAMIE had similar short-term outcomes; besides, RAMIE benefits patients by providing better recurrent laryngeal nerve protection, which suggests RAMIE as a secure and reliable therapeutic choice.

The robotic surgical system offers three-dimensional imaging, up to 10-fold magnification, tremor control, and ambidexterity, which indeed enable a precise dissection of the esophagus and regional lymph node, and a good protection the adjacent structures that need to be preserved. In this way, we can better visualize the correct dissection planes. Therefore, we attributed the better laryngeal nerve protection to the use of RAMIE. To our own experience, the chief surgeon has to keep the arms and body in certain sweatful position for a period of time to finish some procedures in TLMIE. In addition, a recent report showed that $86.9 \%$ surgeons reported symptom or physical discomfort that was attributed to performing TLMIE (19). However, RAMIE allows surgeons to sit comfortably while performing complex procedures during the operation.

Additionally, the robot-assisted technique enables surgeons to perform procedures in tight space. The upper mediastinum and particularly the upper thoracic inlet could be explored with much more ease in RAMIE than the traditional thoracoscopic approach. In contrast, when the instruments of TLMIE reach deep in the thorax, it often causes difficulties because of the fulcrum effect of the ribs. Sometimes, instruments of TLMIE have to approach 
Table 2 Postoperative characteristics compared in matched or unmatched cohorts

\begin{tabular}{|c|c|c|c|c|c|c|}
\hline Variables & \multicolumn{3}{|c|}{ Unmatched cases } & \multicolumn{3}{|c|}{ Matched cases } \\
\hline Pneumonia (\%) & $17(23.0)$ & $12(17.6)$ & 0.432 & $13(24.1)$ & $8(14.8)$ & 0.224 \\
\hline ARDS (\%) & $2(2.7)$ & $3(4.4)$ & 0.670 & $2(3.7)$ & $2(3.7)$ & 1.000 \\
\hline Chylothorax (\%) & $3(4.1)$ & $2(2.9)$ & 1.000 & $2(3.7)$ & $1(1.9)$ & 1.000 \\
\hline Cardiac (\%) & $0(0.0)$ & $2(2.9)$ & 0.228 & $0(0.0)$ & $2(3.7)$ & 0.495 \\
\hline Leakage (\%) & $2(2.7)$ & $6(8.8)$ & 0.153 & $2(3.7)$ & $5(9.3)$ & 0.437 \\
\hline Number of LN & $27.4 \pm 12.8$ & $24.7 \pm 7.5$ & 0.143 & $24.7 \pm 11.2$ & $25.4 \pm 7.5$ & 0.719 \\
\hline Hemorrhage (mL) & $119.7 \pm 90.3$ & $115.7 \pm 73.5$ & 0.774 & $116.5 \pm 85.9$ & $118.9 \pm 77.4$ & 0.879 \\
\hline Mortality (\%) & $0(0.0)$ & $0(0.0)$ & 1.000 & $0(0.0)$ & $0(0.0)$ & 1.000 \\
\hline ICU stay (Day) & $2.6 \pm 3.8$ & $3.8 \pm 5.8$ & 0.175 & $2.5 \pm 3.7$ & $4.0 \pm 6.3$ & 0.138 \\
\hline Hospital stay (Day) & $15.1 \pm 9.5$ & $16.7 \pm 9.3$ & 0.343 & $15.2 \pm 9.8$ & $17.1 \pm 10.1$ & 0.335 \\
\hline Total expenses (KUSD) & $20.2 \pm 9.2$ & $24.9 \pm 8.3$ & 0.002 & $20.8 \pm 9.0$ & $25.3 \pm 9.0$ & 0.009 \\
\hline Expenses per day (KUSD) & $1.4 \pm 0.3$ & $1.7 \pm 0.7$ & 0.002 & $1.5 \pm 0.4$ & $1.7 \pm 0.7$ & 0.028 \\
\hline
\end{tabular}

RAIME, robot-assisted minimally invasive esophagectomy; TLMIE, thoraco-laparoscopic minimally invasive esophagectomy; Cardiac, cardiac complications; Leakage, anastomotic leakage; KUSD, thousand US dollars.

the operative field in a parallel way, which gave rise to difficulties in manoeuvrability, however, the robotic system eliminates these difficulties in manipulation and enable surgeons to do a precise dissection in the thorax cavity (20).

Neo-adjuvant chemoradiotherapy has been demonstrated to be the best therapeutic approach for improving the longterm survival of patients with locally advanced esophageal squamous cell carcinoma (21-23). It is also recommended by The NCCN guideline for esophageal and esophagogastric junction cancers to treat locally advanced squamous tumor (24). Actually, the clinical application of neo-adjuvant chemoradiotherapy needs to take some extra characteristics, such as age, KPS score, patient's subjective intention and tolerance, into considerations. In our study group, a total of 70 patients with clinically stage T1-4N1M0 or T4N0M0 received therapeutic suggestion of neo-adjuvant chemoradiotherapy before treatment. However, only 45 out of the 70 patients eventually undertook neo-adjuvant chemoradiotherapy, while others were quit for higher ages (10 patients aged over 70-year-old), subjective rejection (24 patients) or low KPS score (1 patient). Patients refused to take neo-adjuvant therapy for several reasons. For instance, the chemoradiotherapy had adverse effects, increased expenses, extended the duration of treatment and finally, not all patients would benefit from neo-adjuvant therapy. Therefore, more efforts should be made to improve the effectiveness and reduce the toxicity of neo-adjuvant chemoradiotherapy.

Less hoarseness happened in RAMIE group both before and after matching, which suggested that RAMIE presented better laryngeal nerve protection. However, the incidences of other common postoperative complications were similar in RAMIE and TLMIE group. Weksler et al. reported equivalent impacts of RAMIE and TLMIE on postoperative complications in a retrospective study with 43 patients (14). The inconsistent results on laryngeal nerve injury could be caused by different sample size, which is smaller in the Weksler's study. Furthermore, differences in definitions of complications, different surgical techniques, and the course of neoadjuvant therapy are possible important factors contributing to these discrepancies.

The regional lymph node dissection requires fine splitting of left- and right-paratracheal, left- and rightparaesophageal, and pretracheal lymph node from 
adjacent normal blood vessels, nerves, and organs in the upper thoracic space. Nevertheless, the physiologic course of recurrent laryngeal nerve locates quiet near to the paratracheal and paraesophageal lymph nodes. Therefore, the left- and right-recurrent laryngeal nerve must be exposed and remain visible during the lymph node dissection procedures. The robotic assisted technique provides better laryngeal nerve protections because it has better stereoscopic visions and flexible instruments, which help surgeons to have more delicate dissections of the lymph nodes from adjacent tissues. In contrast, when the instruments of TLMIE reach deep in the upper thorax, the operations could be affected. Thus, the incidence of recurrent laryngeal nerve could be influent by different surgical techniques.

Besides, the incidence of anastomotic leak was lower $(5.6 \%)$ and could be treated conservatively. Usually, the anastomotic leak in the neck would require neck wound reopening and routine sterilization. However, we left a drain tube in neck wound with inner tip beside the anastomosis and external tip connecting a vacuum sealing plastic bottle. The drainage could continuously remove the infectious liquid around the wound. And more importantly, the patients with minor leakage were allowed to swallow 50 to $100 \mathrm{~mL}$ pure water every 3 hours, which helped clear up the inner leakage wound. However, if the patient has severe anastomosis leakage, the surgical treatments such as debridement and suturing or two-stage operations would be necessary.

In comparison with conventional TLMIE, the higher cost is a minor point of RAMIE. Except for the total costs, the cost per day was also calculated to reduce the confounding effect of length of hospital stay. However, both the total expenses and daily expense were significantly higher in RAMIE group. The main reason for the issue is the current monopoly of Intuitive Surgical. Secondly, the social and medical insurance does not cover the cost of RAMIE in China. The solution can be encouraging new competitors to hit the market, which may trigger the development of robotic instruments and help reduce the costs. Additionally, we call for revisions of the Chinese social and medical insurance policy, in order to let more patients receive the benefits of RAMIE.

As a retrospective analysis, this study has its limitations. One of the weaknesses is the existence of selection bias. PSM was applied to balance most of the potential covariates that could post negative effects on the comparisons, in order to draw a more reliable conclusion. Despite the use of PSM, selection bias existed. And this is an early single institute experience.

In conclusion, our study demonstrates the safety and feasibility of RAMIE. The robotic surgical system enables a precise dissection of the mediastinum, which results in good recurrent laryngeal nerve protection. We look forward to a randomized study on the topic, which will be a better way to interpret the potential advantages of RAMIE.

\section{Acknowledgments}

None.

\section{Footnote}

Conflicts of Interest: The authors have no conflicts of interest to declare.

Ethical Statement: The authors are accountable for all aspects of the work in ensuring that questions related to the accuracy or integrity of any part of the work are appropriately investigated and resolved. This study was approved by the institutional review board of Sun Yat-sen University Cancer Center, and the patients' consent was waived.

\section{References}

1. Hulscher JB, van Sandick JW, de Boer AG, et al. Extended transthoracic resection compared with limited transhiatal resection for adenocarcinoma of the esophagus. N Engl J Med 2002;347:1662-9.

2. Boone J, Livestro DP, Elias SG, et al. International survey on esophageal cancer: part I surgical techniques. Dis Esophagus 2009;22:195-202.

3. Yibulayin W, Abulizi S, Lv H, et al. Minimally invasive oesophagectomy versus open esophagectomy for resectable esophageal cancer: a meta-analysis. World J Surg Oncol 2016;14:304.

4. Straatman J, van der Wielen N, Cuesta MA, et al. Minimally Invasive Versus Open Esophageal Resection: Three-year Follow-up of the Previously Reported Randomized Controlled Trial: the TIME Trial. Ann Surg 2017;266:232-6.

5. Biere SS, van Berge Henegouwen MI, Maas KW, et al. Minimally invasive versus open oesophagectomy for patients with oesophageal cancer: a multicentre, open-label, randomised controlled trial. Lancet 2012;379:1887-92. 
6. Luketich JD, Pennathur A, Awais O, et al. Outcomes after minimally invasive esophagectomy: review of over 1000 patients. Ann Surg 2012;256:95-103.

7. Lv L, Hu W, Ren Y, et al. Minimally invasive esophagectomy versus open esophagectomy for esophageal cancer: a meta-analysis. Onco Targets Ther 2016;9:6751-62.

8. Guo W, Ma X, Yang S, et al. Combined thoracoscopiclaparoscopic esophagectomy versus open esophagectomy: a meta-analysis of outcomes. Surg Endosc 2016;30:3873-81.

9. Yerokun BA, Sun Z, Jeffrey Yang CF, et al. Minimally Invasive Versus Open Esophagectomy for Esophageal Cancer: A Population-Based Analysis. Ann Thorac Surg 2016;102:416-23.

10. van Hillegersberg R, Boone J, Draaisma WA, et al. First experience with robot-assisted thoracoscopic esophagolymphadenectomy for esophageal cancer. Surg Endosc 2006;20:1435-9.

11. Boone J, Schipper ME, Moojen WA, et al. Robot-assisted thoracoscopic oesophagectomy for cancer. Br J Surg 2009;96:878-86.

12. Park SY, Kim DJ, Yu WS, et al. Robot-assisted thoracoscopic esophagectomy with extensive mediastinal lymphadenectomy: experience with 114 consecutive patients with intrathoracic esophageal cancer. Dis Esophagus 2016;29:326-32.

13. Galvani CA, Gorodner MV, Moser F, et al. Robotically assisted laparoscopic transhiatal esophagectomy. Surg Endosc 2008;22:188-95.

14. Weksler B, Sharma P, Moudgill N, et al. Robot-assisted minimally invasive esophagectomy is equivalent to thoracoscopic minimally invasive esophagectomy. Dis Esophagus 2012;25:403-9.

15. Kim DJ, Hyung WJ, Lee CY, et al. Thoracoscopic esophagectomy for esophageal cancer: feasibility and safety of robotic assistance in the prone position. J Thorac Cardiovasc Surg 2010;139:53-9.e1.

16. van Hillegersberg R, Seesing MF, Brenkman HJ, et al. Robot-assisted minimally invasive esophagectomy. Chirurg
2017;88:7-11.

17. Tan Z, Zhang X, Wang X, et al. Short-term outcomes of robot-assisted minimally invasive esophagectomy for esophageal cancer. Zhonghua Wei Chang Wai Ke Za Zhi 2016;19:995-8.

18. McMurry TL, Hu Y, Blackstone EH, et al. Propensity scores: Methods, considerations, and applications in the Journal of Thoracic and Cardiovascular Surgery. J Thorac Cardiovasc Surg 2015;150:14-9.

19. Park A, Lee G, Seagull FJ, et al. Patients benefit while surgeons suffer: an impending epidemic. J Am Coll Surg 2010;210:306-13.

20. Kim DJ, Park SY, Lee S, et al. Feasibility of a robotassisted thoracoscopic lymphadenectomy along the recurrent laryngeal nerves in radical esophagectomy for esophageal squamous carcinoma. Surg Endosc 2014;28:1866-73.

21. Yang H, Liu H, Chen Y, et al. Neoadjuvant Chemoradiotherapy Followed by Surgery Versus Surgery Alone for Locally Advanced Squamous Cell Carcinoma of the Esophagus (NEOCRTEC5010): A Phase III Multicenter, Randomized, Open-Label Clinical Trial. J Clin Oncol 2018;36:2796-803.

22. Chao YK, Hsieh MJ, Liu YH, et al. Lymph Node Evaluation in Robot-Assisted Versus Video-Assisted Thoracoscopic Esophagectomy for Esophageal Squamous Cell Carcinoma: A Propensity-Matched Analysis. World J Surg 2018;42:590-8.

23. Shapiro J, van Lanschot JJ, Hulshof MC, et al. Neoadjuvant chemoradiotherapy plus surgery versus surgery alone for oesophageal or junctional cancer (CROSS): long-term results of a randomised controlled trial. Lancet Oncol 2015;16:1090-8.

24. Network NCC. Esophageal and Esophagogastric Junction Cancers (Version 1.2019). Available online: https://www. nccn.org/professionals/physician_gls/pdf/esophageal.pdf. Accessed August 12, 2019.
Cite this article as: Chen J, Liu Q, Zhang X, Yang H, Tan Z, Lin Y, Fu J. Comparisons of short-term outcomes between robot-assisted and thoraco-laparoscopic esophagectomy with extended two-field lymph node dissection for resectable thoracic esophageal squamous cell carcinoma. J Thorac Dis 2019;11(9):3874-3880. doi: 10.21037/jtd.2019.09.05 